\title{
ARTículos
}

\section{Mediciones confiables para el cuidado de la salud humana y la preservación del ambiente ante la exposición a nanomateriales}

\author{
Rubén J. Lazos Martínez*
}

\begin{abstract}
RESUMEN: Se consideran como vertientes en el desarrollo de las nanotecnologías las que se refieren a los productos novedosos que propician la competitividad de las economías, y las que tratan de sus consecuencias para la sociedad, en particular, de los efectos en la salud humana y en el ambiente por la exposición a nanomateriales, cuyos avances presentan un rezago considerable respecto a la primera vertiente; se dispone de resultados concluyentes sólo en unos pocos casos. Como respaldo de tales conclusiones hacen falta mediciones confiables cuyas características de trazabilidad metrológica y de su incertidumbre de medida son discutidas.

PALABRAS Clave: nanotecnología, metrología, salud humana, ambiente.
\end{abstract}

AвSTRACT: Two axes regarding the developpment of nanotechnologies are considered, that dealing with new products that fosters the competitiveness of the economies, and the one referring to the consequences on the society, particularly the effects on the human health and the environment, advance slower than the first one; concluding results are available in only very few cases. In order to support those, reliable measurements with valid metrological traceability and measurement uncertainty are required.

KEYWORDS: nanotechnology, metrology, human health, environment.

\section{INTRODUCCIÓN}

La nanotecnología nos ha alcanzado: se encuentran con facilidad en México pantalones repelentes a las manchas, recipientes para la conservación de alimentos por varias semanas, lavadoras con bactericidas, cosméticos y bloqueadores solares muy eficaces para la protección de la piel, cementos súper resistentes, productos cuyas novedosas características son pretendidamente debidas a sus contenidos de nanomateriales. Incluso, son mucho más sorprendentes los logros en otras disciplinas que no hubieran podido ser alcanzados sin los nanomateriales, por ejemplo, la regeneración de nervios, la entrega precisa de fármacos en las células pertinentes, la remediación de aguas contaminadas, entre otros muchos.

Para los fines de este trabajo, el término "nanomaterial" se utiliza para referirse genéricamente a cualquier sustancia conteniendo alguna forma de material con características propias de la nanoescala.

La importancia creciente de las nanotecnologías puede percibirse en, por ejemplo, la velocidad de crecimiento de los negocios: en 1994, la inversión global en nanotecnologías se estimaba aproximadamente en $€ 5$ billones (Royal, 2004: 1), mientras que la producción manufacturera global asciende una década después a US\$ 2.6 tri-

* Centro Nacional de Metrología, México. (rlazos@cenam.mx) 
llones (Friedrichs, 2007). De hecho, el número de productos al consumidor con base en nanomateriales se ha casi cuadruplicado en los últimos 4 años (PEN, 2010) superando el millar en 2009; es notable que entre ellos los productos relacionados con la salud y la belleza humanas hayan ocupado el primer lugar por su número a lo largo de varios años. Por otro lado, el gobierno de los EUA, después de una inversión inicial de US M\$500, en diez años, ha hecho inversiones acumuladas de US \$12 billones (Holdren, 2010).

Por otro lado, las actividades de investigación y desarrollo en nanociencias y nanotecnologías - NyN - se han acelerado de forma muy importante, baste mencionar que el número de patentes creció al cuádruplo en seis años (de 531 en 1995 a 1976 en 2001) (Royal, 2004) y que el número de publicaciones en el tema, por lo menos se triplicó de 1994 a 2004 (CIMAV, 2007). Cabe anotar que la denominación de nanociencia o nanotecnología es relativamente reciente y que ha englobado a disciplinas con una ya larga evolución previa, siendo ejemplo de ello el estudio de películas delgadas en materiales semiconductores.

Adicionalmente, como previsión para atender el acelerado crecimiento de las NyN, en razón de que se consideran insuficientes los recursos humanos formados en la materia, y para mantener su posición como país en el desarrollo de las NyN, se está recomendando al gobierno de los EUA propiciar la permanencia en ese país de los recursos humanos formados en el mismo (Holdren, 2010).

\section{Efectos en la SALUd humana y en EL AMBIENTE}

Como en toda tecnología novedosa, junto a la aparición de los nuevos productos que conlleva, se encuentran los riesgos de la exposición en alguna de sus formas de los seres humanos a los nanomateriales, y del impacto de éstos en el ambiente en alguna fase de su ciclo de vida. Se entiende que los nuevos productos están asociados a la competitividad de un país, que ellos propiciarían cierto empuje económico. La consideración a los riesgos mencionados tendería a la conservación de la salud humana y del ambiente, aspecto de no menor importancia para la sociedad dado que es uno de los elementos que soporta la sustentabilidad de las nanotecnologías.

Es común entre los estudiosos del tema considerar que los factores de riesgo son la exposición a un nanomaterial y la peligrosidad o toxicidad de dicho material.

Grandes recursos se están dedicando al estudio de los efectos de los nanomateriales en los seres humanos y el ambiente. Se han identificado 158 estudios sobre el tema completos o en su fase terminal en el periodo 2004-2009, y otros 119 por terminarse en fechas posteriores (Aitken, 2009). La mayor parte de ellos están orientados a la salud humana; sin embargo, se han emprendido muchas menos investigaciones sobre los tópicos ambiente y caracterización.

Una de las mayores inquietudes en los países que albergan plantas industriales en las cuales se producen o se manejan nanomateriales, y donde a menudo se utiliza tecnología importada, radica en que la mano de obra local está sujeta a riesgo por la exposición a nanomateriales por lapsos tan prolongados como su jornada laboral aunque debe reconocerse que la mayor parte de los nanomateriales están embebidos en estructuras que les impiden moverse libremente. No sobra resaltar que además de los efectos a corto plazo de la exposición, deben considerarse aspectos epidemiológicos, de persistencia y de bioacumulación. 
En operarios expuestos a nanopartículas por inhalación, se estima que el 50\% de las partículas con tamaño de alrededor de $20 \mathrm{~nm}$ se depositan en la región alveolar (ver, por ejemplo, ISO 12885, 2008: 23) y que aquéllas con baja solubilidad dan lugar a enfermedades pulmonares (Aitken, 2004). Los efectos en la salud humana de la exposición a nanopartículas no solamente dependen de su masa, sino de su área superficial - a menor tamaño mayor toxicidad-, y de su estado de agregación. En otro caso, aún cuando se ha encontrado que las nanopartículas de dióxido de titanio usadas en filtros solares para el cuidado de la piel no penetran en la piel sana, no es claro que dicho resultado prevalezca cuando se trata de pieles con alguna clase de daño o que sufran alteraciones leves como eczemas (véase, por ejemplo, Royal, 2004). Adicionalmente, se ha reportado la translocación de nanopartículas a diferentes órganos en el cuerpo una vez que han penetrado el epitelio celular.

Los nanotubos de carbono - NTC - constituyen otros nanomateriales de carbono con una muy amplia variedad de aplicaciones. Se encuentran con diversas geometrías — diámetro y longitud—, número de paredes e impurezas, resultantes de su método de producción. Se presentan con un aspecto fibroso, similar a las fibras de asbesto, pero a diferencia de éstas, generalmente se agrupan en "gavillas" entrelazadas. Los resultados de los estudios son sensibles a las características del material usado en la prueba, por lo que es importante precisarlas con el detalle suficiente. Por ejemplo, se encuentra que las NTC purificados con ácido han producido en ratas inflamación, fibrosis y granulomas en los pulmones después de una única aspiración. Asimismo, hay evidencias de que los NTC con más de $20 \%$ en peso de impurezas de hierro inducen inflamaciones pulmonares más severas que sus contrapartes purificados (ISO 12885, 2008).

Sobre el uso de los resultados de estudios para obtener conclusiones confiables, cabe anotar algunos argumentos que mueven a su cuestionamiento [Grainger, 2010], entre los cuales se encuentran: la falta de una caracterización confiable o completa de los nanomateriales bajo prueba, la interferencia del nanomaterial con el ensayo, los cambios del estado físico del nanomaterial — como estado de agregación- dentro del sistema biológico, la falta de reproducibilidad de resultados entre laboratorios, la posible sobre-simplificación del modelo de toxicidad, la no necesariamente presente correlación directa entre resultados in vitro-in vivo.

Dada la complejidad de los sistemas biológicos como el ser humano, o los que están integrados en el ambiente, no es sorprendente la dificultad para realizar estudios definitorios y concluyentes sobre los efectos de los nanomateriales en la salud humana y en el ambiente.

Desde la perspectiva del consumidor final, es notable que, en general, la publicidad sobre el contenido nano esté sufriendo cierto decremento después de haber mostrado un aumento notable (ver, por ejemplo, Rogers, 2005), aparentemente debido a la percepción por parte de la sociedad de que los nanomateriales son de alguna manera nocivos para la salud humana; la falta de resultados sólidos concluyentes ha dado lugar a especulaciones.

A manera de resumen sobre los efectos de la exposición a nanomateriales, actualmente parece haber esperanzas de que la mayoría de ellos no sean nocivos para la salud humana ni para el ambiente, pero se encuentran indicaciones de que la exposición a algunos conlleva riesgos relevantes. Por tanto, es indispensable continuar o emprender los estudios correspondientes para confirmar dichas esperanzas o indica- 
ciones con evidencias más sólidas, no solamente sobre la existencia o no de efectos nocivos sino de la cuantificación del nivel de exposición a partir del cual se presentan dichos efectos -la especificación.

\section{Mediciones para las NyN}

Las medidas, como resultantes de procesos de medición, constituyen una de las formas que como comunidad hemos aprendido a utilizar para dar objetividad y solidez a nuestros hallazgos; de hecho, de acuerdo con el método científico, las hipótesis se confirman, se modifican o se desechan con base en resultados de medición. Para que las mediciones sean confiables deben producir resultados equivalentes, aunque no necesariamente iguales, independientes del lugar, del momento, de la persona que las lleve a cabo, y hasta del método utilizado, siempre y cuando la magnitud sujeta a medición —el mensurando [VIM, 2009] — sea la misma. Debe notarse que un mensurando, en tanto sujeto a medición, es cuantificable por definición.

La confiabilidad de las medidas se traduce en los conceptos de trazabilidad metrológica e incertidumbre de la medida.

La trazabilidad metrológica es la propiedad de las medidas por la cual se relacionan a una referencia determinada (VIM, 2009), la cual les confiere la característica de producir resultados reproducibles como se ha mencionado. Cabe anotar que la referencia determinada es en última instancia la realización de la definición de la unidad de medida correspondiente. Por ejemplo, se espera que el valor del tamaño de las nanopartículas esté relacionado con la definición del metro como unidad de longitud en el sistema internacional (BIPM, 2006), y, si los diversos interesados toman la misma referencia para las mediciones del tamaño de partícula de la misma muestra, los resultados que obtengan por separado coincidirán dentro de un cierto intervalo de valores previsible, denominado incertidumbre de la medida.

Desafortunadamente estos dos conceptos, trazabilidad metrológica e incertidumbre de la medida, a menudo pasan desapercibidos cuando se generan los métodos de medición y caracterización de nanomateriales, aun en el ámbito de la normalización internacional en la materia. Se reconocen algunas dificultades para medir algunas características, ya sea porque:

a. No ha podido encontrarse una forma de establecer la relación con la unidad, por ejemplo, las mediciones de longitud del orden de algunos nanómetros no han terminado de relacionarse en toda la extensión con la unidad de longitud (Wilkening, 2005).

b. Aún no ha sido posible definir las unidades de medida de algunas magnitudes en términos de unidades definidas por consenso, aún cuando no sean las del sistema internacional, como la exposición, por su dependencia de varios factores, algunos todavía no determinados con certeza.

c. En un considerable número de casos no se cuenta con un modelo de medición como requiere la estimación de la incertidumbre de medida, a partir del cual hacer las cuantificaciones estadísticas necesarias.

d. No se ha podido traducir la característica de interés como un atributo a una o varias variables medibles, a mensurandos. 
Respecto a esta última dificultad, en el comité técnico ISO TC229 Nanotechnologies el grupo sobre ambiente, salud y seguridad, responsable de recomendar especificaciones sobre nanomateriales en atención a estos rubros, ha tenido que tender enlaces con el grupo de medición y caracterización del mismo comité para definir apropiadamente los mensurandos cuyos métodos de medición puedan ser desarrollados o recomendados por parte de éste (Height-Walker, 2010).

Se cuenta con varias formas para conseguir que una medida sea metrológicamente trazable:

a. Por comparación con una referencia metrológica — un patrón de medida-, a su vez metrológicamente trazable a otra referencia metrológicamente trazable —operación denominada calibración-, hasta llegar a una realización de la definición de la unidad.

b. Por el uso de un material de referencia certificado, material suficientemente homogéneo y estable con respecto a propiedades determinadas, acompañado por un documento que avale los valores e incertidumbres obtenidos por un procedimiento reconocido como válido (VIM, 2009).

c. Por la aplicación rigurosa de un método acordado por la comunidad internacional.

Los resultados de medición constituyen uno de los ingredientes vitales en la toma de decisiones, pues soportan primero la determinación de especificaciones y, en etapas posteriores, proveen de información para comparar con dichas especificaciones — valores límite permitidos para una magnitud dada.

No es trivial el proceso de determinar especificaciones, y en particular a partir de la identificación de un riesgo para la salud humana o para el ambiente. La validez del proceso completo depende de la solidez de los hallazgos que se encuentren para cada etapa. El proceso tampoco es lineal como pudiera a primera vista interpretarse del esquema en la figura 1, contiene múltiples ciclos de realimentación en cada etapa y a lo largo del mismo, por ejemplo, la determinación de las especificaciones en la última etapa requiere de los resultados sobre los niveles de exposición y toxicidad permitidos que se encuentren en la primera etapa, una vez que se disponga de métodos válidos de medición. Además, las etapas difícilmente son llevadas a cabo por la misma organización; es frecuente y deseable que en cada etapa concurran los esfuerzos de instituciones varias.

FIGURA 1. Esquema del proceso para determinar especificaciones a partir de la identificación de un riesgo. El rombo y la flecha bidireccional indican una verificación. Cabe anotar que para fines de claridad se muestra una versión enormemente simplificada del proceso.

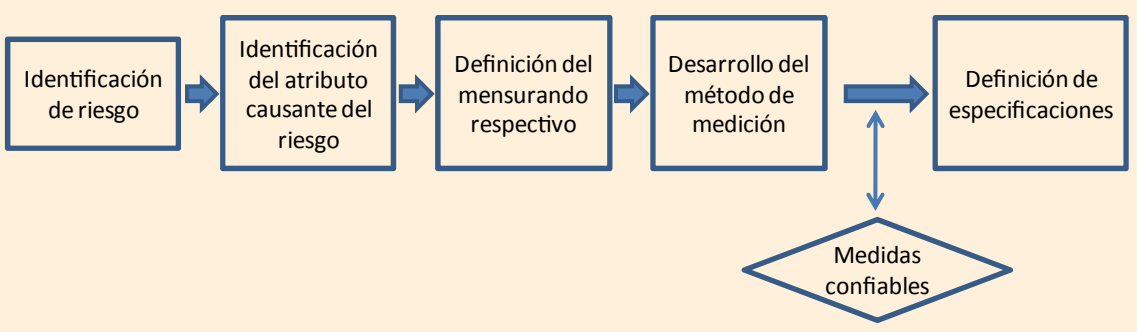




\section{¿QUÉ MEDIR PARA LAS NANOTECNOLOGÍAs?}

En el contexto de los efectos sobre la salud humana y el ambiente, hay coincidencia en que las necesidades elementales de medición relativas a nanopartículas en el aire incluyen la detección de las mismas, determinar si están presentes o no, y proceder entonces a medir su tamaño y la distribución de su tamaño, su concentración, su área superficial, así como a caracterizar su estado de agregación. Por supuesto, queda una larga lista de propiedades que atender, y cuya prioridad depende del nanomaterial de que se trate. Una lista similar aplica para NTC, y los otros nanomateriales.

En la literatura se encuentran numerosos métodos y técnicas para la medición o caracterización de nanopartículas y de NTC (ver, por ejemplo, Hassellov, 2008 o Lojkowski, 2006), entre los que se encuentran, microbalanzas en conjunto con selectores de tamaño de partícula para masa, contadores de partículas por condensación, microscopía electrónica de transmisión o de barrido para la determinación de geometría y morfología, entre otros. Desafortunadamente, muchos de estos métodos o técnicas adolecen de alguna deficiencia que les impide producir resultados de medición confiables en el sentido comentado en este trabajo.

En particular, la inexistencia de referencias metrológicas es una situación generalizada en el ámbito internacional, no privativa de nuestro país, de modo que la comunidad internacional está haciendo esfuerzos para aliviarla mediante tres vías principales:

a. La elaboración de materiales de referencia certificados, para la cual se ha logrado conjuntar la información en un catálogo público (COMAR).

b. La oferta de servicios de calibración confiables que desafortunadamente es aún muy escasa, tan sólo el National Physical Laboratory del Reino Unido ofrece la calibración de contadores de partículas por condensación.

c. La implementación del Arreglo de Reconocimiento Mutuo del Comité Internacional de Pesas y Medidas, como resultado de la cual se ha generado una base de datos de servicios ofrecidos por los laboratorios nacionales de metrología a todo interesado, evaluados por sus pares (KCDB). Debe mencionarse que actualmente esta base no contiene tantos servicios orientados a las nanotecnologías como se espera en un futuro cercano.

En México, el Centro Nacional de Metrología desde hace varios años ha emprendido actividades tendientes a satisfacer algunas de las necesidades de medición para las nanotecnologías, enfocadas a proveer de referencias determinadas para implementar o fortalecer la trazabilidad metrológica de las mediciones que para las nanotecnologías se realicen en el país.

Entre tales actividades se encuentran:

a. La producción de materiales de referencia para tamaño de partícula inscritos en COMAR.

b. Participaciones en pruebas de comparación entre laboratorios sobre determinación de tamaño de partícula, promovidas por el Programa de metrología Asia-Pacífico (APMP) y por el Versailles Project on Advanced Materials and Standards (VAMAS), donde se ha demostrado que los resultados producidos en la materia son confiables. 
c. La participación en el Arreglo de Reconocimiento Mutuo del Comité Internacional de Pesas y Medidas desde su origen, en donde aún no están incluidas entre nuestras capacidades de medición o calibración inscritas en el Arreglo, las dirigidas a las nanotecnologías.

\section{¿Qué POdemos hacer en MÉxico Para enfrentar los Retos DE LAS NANOTECNOLOGÍA?}

- Acelerar el desarrollo de patrones de medida y métodos de medición para la caracterización confiable de productos con base en naomateriales y del ambiente laboral particularmente.

- Incentivar la investigación científica y el desarrollo tecnológico en la materia.

- Elaborar sinérgicamente por parte de los involucrados y promulgar por parte de las autoridades competentes, un marco normativo que prevenga o mitigue los efectos en la salud humana y el ambiente de la exposición a nanomateriales.

- Aplicar a las nanopartículas libres y nanotubos de carbono el mismo trato de manejo precavido que se aplica a nuevos productos químicos (Royal, 2004).

- Recomendar a los fabricantes la emisión de una declaración formal de los nanomateriales que se hayan agregado a sus productos.

- Publicar por parte de los fabricantes los procedimientos para el manejo de los productos con nanopartículas y nanotubos de carbono en las fases finales de su ciclo de vida, ya sea vía desecho, destrucción o reciclado (Royal, 2004).

- Promover la participación de México en los diversos foros internacionales, como la OCDE o ISO, para incorporar los puntos de vista nacionales en las decisiones de carácter global al respecto.

\section{Conclusiones}

Los estudios de los efectos no deseables en la salud humana y el ambiente a consecuencia de la exposición a nanomateriales aún no han producido resultados concluyentes, causa de inquietudes para la sociedad en su necesidad de asegurar la sustentabilidad de las nanotecnologías.

Los resultados de medición confiables producen certeza en las conclusiones y en la toma de decisiones, su confiabilidad depende de que presenten la propiedad de trazabilidad metrológica a una referencia válida y de una estimación apropiada de su incertidumbre de medida. El camino para lograr la obtención de medidas confiables para atender las nanotecnologías aún es largo, sin embargo México, en concierto con la comunidad internacional, procura acelerar el paso dentro de sus posibilidades.

\section{RefERENCIAS}

Aitken R. J., K. S. Creely, C. L. Tran. 2004. Nanoparticles: An occupational hygiene review. Research Report 274, Institute of Occupational Medicine, for the Health and Safety Executive.

Aitken R. J., S. M. Hankin, B Ross, C. L. Tran, V. Stone, T. F. Fernandes, K. Donaldson, R. Duffin, Q. Chaudhry, T. A. Wilkins, S. A. Wilkins6, L. S. Levy, S. A. Rocks, A. Maynard. 2009. 
EMERGNANO: A review of completed and near completed environment, health and safety research on nanomaterials and nanotechnology. Defra Project CB0409 Report TM/09/01, marzo.

APMP, Asia-Pacific Metrology Programme. Información en http://www.apmpweb.org/ BIPM, Bureau international des Poids et Measures. 2006. The international system of units, 8a. ed. p. 112. Disponible en http://www.bipm.org/en/si/si_brochure/general. html.

CIMAV. 2008. Diagnóstico y prospectiva de la nanotecnologia en Mexico. Febrero.

KCDB, The BIPM Key Comparison Database. Disponible para consulta en http://www.bipm. org/ .

COMAR, The international database for certified reference materials. Disponible para consulta en http://www.comar.bam.de/en/ .

Friedrichs S., Schulte J., 2007. Science and Technology of Advanced Materials, 8, 12 -18.

Grainger David W. 2010. Challenges facing in vitro nanotoxicity assays:analysis, dispersion, dosimetry, and answer. 4th. Tri-national Workshop on Standards for Nanotechnology, Ottawa. Disponible en http://www.nrc-cnrc.gc.ca/eng/events/inms/2010/02/03/ tri-national-workshop.html .

Hassellov M., Readman J. W., Ranville J. F. and Tiede K. 2008. "Nanoparticle analysis and characterization methodologies in environmental risk assessment of engineered nanoparticles". Ecotoxicology 17:344-361.

Height-Walker A. 2010. ISO TC229 Joint Task Group: understanding measurands for nano EHS comparability, 4th. Tri-national Workshop on Standards for Nanotechnology, Ottawa. Disponible en http://www.nrc-cnrc.gc.ca/eng/events/inms/2010/02/03/ tri-national-workshop.html

Holdren J. P. et al. 2010. Report to the President and Congress on the Third Assessment of the National Nanotechnology Initiative, marzo.

ISO TR 12885. 2008. Nanotechnologies - Health and safety practices in occupational settings relevant to nanotechnologies. Este documento hace referencia a los trabajos originales que soportan sus afirmaciones.

Lojkowski W., Turan R., Proykova A., Daniszewska A. (eds). 2006. Eight Nanoforum Report: Nanometrology. julio. Disponible en www.nanoforum.org.

PEN, The Project on Emerging Nanotechnologies. 2010. Consulta en www.nanotechproject.org/inventories/consumer/updates/ el 12 de abril de 2010.

Rogers L. 2005. Safety fears over 'nano' anti-ageing cosmetics. The Sunday Times, julio 17.

Royal Society (The) and the Royal Academy of Engineering. 2004. Nanoscience and nanotechnologies, julio.

VAMAS, Versailles Project on Advanced Materials and Standards. Disponible para consulta en http://www.vamas.org/ .

VIM. 2008. Vocabulario Internacional de Metrología. Conceptos fundamentales y generales, y términos asociados. JCGM 200:2008. BIPM, IEC, IFCC, ILAC, ISO, IUPAC, IUPAP, OIML. 2009. 2009. Norma mexicana NMX-Z-055-2009-IMNC, equivalente al original en inglés en su traducción al español. Disponible en www.cenam.mx.

Wilkening G. and Koenders L. (eds.) 2005. Nanoscale calibration standards and methods. Wiley-VCH. 